\title{
First-principles study of sulfur atom doping and adsorption on
}

\section{$\alpha-\mathrm{Fe}_{2} \mathrm{O}_{3}(0001)$ film}

\author{
Jiao $\mathrm{An}^{1}$, Prabath Wanaguru ${ }^{1}$, Congxin Xia ${ }^{1,2}$, Meng Tao ${ }^{3}$, and Qiming Zhang ${ }^{1}$ \\ 1. Department of Physics, the University of Texas at Arlington, Arlington, TX 76019, USA. \\ 2. College of Physics and Electronic Engineering, Henan Normal University, Xinxiang, Henan \\ 453007, China.
}

3. School of Electrical, Computer and Energy Engineering Laboratory for Terawatt Photovoltaics, Arizona State University, Tempe, AZ 85287, USA

\begin{abstract}
Using the spin-polarized density functional theory (DFT) and the DFT $+U$ method, the geometric and electronic properties of the hematite $\alpha-\mathrm{Fe}_{2} \mathrm{O}_{3}(0001)$ film with the sulfur (S) atom doping and adsorption have been investigated systematically. The most stable hematite $\alpha-\mathrm{Fe}_{2} \mathrm{O}_{3}(0001)$ film with an anti-ferromagnetic arrangement is identified. For the study of sulfur adsorption on the film, the $\mathrm{S}$ adatom prefers to bond with three $\mathrm{O}$ atoms, in the center of a triangle formed by the three $\mathrm{O}$ atoms. The $\mathrm{S}$ acts as a cation at this site. The sulfur adsorption has introduced two gap states, in addition to the unoccupied surface states. Furthermore, with the most stable S-adsorption configuration, the diffusion of the $\mathrm{S}$ adatom from the surface to the inside is searched and the transition state along the minimum-energy pathway is also evaluated. For S-doping in the film, it is found that $\mathrm{S}$ substitution of $\mathrm{O}$ in the top layer is energetically favored than that in the deeper layer. It shows that the value of the band gap is reduced to $\sim 1.26 \mathrm{eV}$ from $\sim 1.43 \mathrm{eV}$ of the clean film. The formation energy of $\mathrm{S}$ substitution of $\mathrm{O}$ in the film is also obtained.
\end{abstract}

Keywords: $\alpha-\mathrm{Fe}_{2} \mathrm{O}_{3}$ (0001) film; Sulfur adsorption; Isovalent doping

* Corresponding author: Tel: 1-817-272-2020; Fax: 1-817-272-3637.

E-mail: zhang@uta.edu 


\section{Introduction}

Transition metal oxides have drawn a lot of interest in both experimental and theoretical research due to their unique physical and chemical properties. As an important member of them, hematite $\alpha-\mathrm{Fe}_{2} \mathrm{O}_{3}$ has been investigated for its potential use in a wide range of applications, such as catalysis, electrochemistry, magnetization, and environmental applications [1-4]. The various properties of the bulk and surface hematite have been studied extensively. It includes the studies on the crystal structures $[5,6]$, the electrical properties of the bulk [7] and its surface [8], and the magnetic properties $[9,10]$. Moreover the optical properties of bulk and thin film $\alpha-\mathrm{Fe}_{2} \mathrm{O}_{3}$ have been analyzed experimentally $[11,12]$.

Recently hematite $\alpha-\mathrm{Fe}_{2} \mathrm{O}_{3}$ has drawn extensive interest due to its potential application in solar water splitting and solar energy conversion [13]. It is a cost-effective, earth-abundant and environment-friendly material with a favorable band gap $(\sim 2.1 \mathrm{eV})[14]$. These are necessary requirements for massive applications in the solar energy conversion at an affordable cost. To improve its property as photo-electrode material, M-doped $\alpha-\mathrm{Fe}_{2} \mathrm{O}_{3}$ film $(\mathrm{M}=\mathrm{Cr}, \mathrm{Mo}, \mathrm{Pt}, \mathrm{Ti})$ has been studied experimentally [15-18]. The first-principles studies on Co and Ni doped hematite film have also been conducted [19]. They exhibit the higher photoelectrochemical (PEC) performance than that of the undoped samples. As the application of the photovoltaic (PV) material, however, it has been found that the potentially low-cost material does not have satisfactory performance in practice, which could be attributed to the 
relatively poor absorptivity and indirect band gap [11, 20]. To modify the electronic structures and transport properties of $\alpha-\mathrm{Fe}_{2} \mathrm{O}_{3}$, the hematite doped with $\mathrm{Al}, \mathrm{Ti}$, and $\mathrm{Cu}$ have been investigated experimentally and theoretically [21-23]. But no improvement in the performance has been achieved for hematite in solar energy conversion. It is well known that a direct band gap ranging from $1.30 \mathrm{eV}$ to $1.60 \mathrm{eV}$ is optimal for a solar cell material. The influence of different concentrations of isovalent sulfur (S) doping in the bulk hematite on the band structure and optical absorption has recently been investigated [24]. It elucidates that the $\alpha-\mathrm{Fe}_{2} \mathrm{O}_{3-x} \mathrm{~S}_{x}$ with $\mathrm{S}$ concentration of $\sim 5.6 \%$ has a direct band gap with a desirable value $(\sim 1.45 \mathrm{eV})$.

Hence, it is worth to further investigate the incorporation of $\mathrm{S}$ atom into $\alpha-\mathrm{Fe}_{2} \mathrm{O}_{3}$. As an initial stage, the adsorption and substitution of $\mathrm{S}$ atom at the surface of $\alpha-\mathrm{Fe}_{2} \mathrm{O}_{3}$ need to be understood. Firstly, a surface model should be obtained. The properties of the clean hematite $\alpha-\mathrm{Fe}_{2} \mathrm{O}_{3}$ films have already been investigated. The electronic structure of the clean hematite $\alpha-\mathrm{Fe}_{2} \mathrm{O}_{3}$ (0001) surface has been discussed theoretically in detail by W. Bergermayer et al. [25]. Under low and intermediate oxygen pressures the Fe-terminated surface is most stable. But under high oxygen pressure the $\mathrm{O}_{3}$-terminated surface is more stable. The electronic properties of the graphene-hematite interfaces have been studied [26]. The interaction mechanism between $\mathrm{H}_{2} \mathrm{~S}$ and the $\alpha-\mathrm{Fe}_{2} \mathrm{O}_{3}(0001)$ surface [27] was studied by density functional theory (DFT) in order to remove $\mathrm{H}_{2} \mathrm{~S}$ from coal gas. Some recent studies by DFT also considered different metal atoms, i.e. $\mathrm{Mg}, \mathrm{Au}$ and $\mathrm{Pb}$, adsorption on the $\alpha-\mathrm{Fe}_{2} \mathrm{O}_{3}$ (0001) surface [28, 29]. 
In the present work, a film model with (0001) surface of $\alpha-\mathrm{Fe}_{2} \mathrm{O}_{3}$ is studied by the DFT $+U$ method. Then the $\mathrm{S}$ adsorption on the surface and the isovalent $\mathrm{S}$ doping in the film are investigated. The computational details are given in the section 2 . The main results are presented and discussed in Section 3. The results provide a better understanding of the initial stage of the sulfuration of hematite iron oxide.

\section{Computational details and models}

All the calculations performed in this work are based on spin-polarized DFT as implemented in the Vienna $a b$ initio simulation package (VASP) [30, 31]. The exchange-correlation energy is treated within the generalized gradient approximation (GGA) functional and parameterized by Perdew-Burke-Ernzerhofer (PBE) [32] functional. The electron-ion interaction is described by the projector augmented wave (PAW) potentials [33], treating Fe $3 p, 3 d$ and $4 s, O 2 s$ and $2 p$, and $S 3 s$ and $3 p$ states as valence states. A plane wave basis with an energy cutoff of $400 \mathrm{eV}$ is used and the convergence for energy is chosen as $10^{-5} \mathrm{eV}$. In order to describe the effect of the on-site coulomb repulsion of Fe-3d electrons more accurately, the exchange-correlation energy is treated by the GGA $+U$ approach used by Dudarev et al.[34] with $U=4 \mathrm{eV}$. Full structural optimization is carried out until the force converged below $0.05 \mathrm{eV} / \AA$. The search for the transition states along the minimum-energy pathway between two stable configurations is calculated using the climbing-image nudged elastic band (CI-NEB) method [35]. There are eight images used in the calculation.

To demostrate the accuracy of our computation setting, the structure of bulk 
hematite with a hexagonal prime cell consisting of six formula units is calculated first. Integrations over the first Brillouin zone (FBZ) are performed with a $5 \times 5 \times 5$ Monkhorst-Pack [36] k-point grid. All possiple magnetic phases are considered. The global energy minimum state is an anti-ferromagnetic (AFM) phase with magnetic moment of $4.15 \mu_{\mathrm{B}}$ on each $\mathrm{Fe}$ atom. The optimized lattice constants of the bulk $\alpha-\mathrm{Fe}_{2} \mathrm{O}_{3}(\mathrm{a}=5.043 \AA, \mathrm{c}=13.729 \AA)$ are in excellent agreement with experimental values ( $\mathrm{a}=5.035 \AA, \mathrm{c}=13.747 \AA)$ [5]. It has an indirect band gap with the value of 2.05 $\mathrm{eV}$, in good agreement with the experimental and the previous results [5].

The hematite (0001) surface is a stable surface at oxygen partial pressure and ambient temperature [25]. The present work will focus on this surface. The (0001) surface is modeled by a thin film built of 12 atomic layers and a vacuum region of 15.0 $\AA$ normal to the film in a periodic boundary condition as shown in Fig. 1. In order to keep the stoichiometry for simplicity, there are two possible films for consideration. The film of type A is that one surface is terminated by Fe atoms while another surface is terminated by $\mathrm{O}$ atoms, as shown in Fig. 1(a). The film type B is that both sides of the surface are terminated by Fe atoms, as shown in Fig. 1(b). The FBZ of the $1 \times 1 \times 1$ surface unit cell is sampled by $5 \times 5 \times 1 k$-points. While for the $2 \times 2 \times 1$ surface supercell, a $3 \times 3 \times 1 \mathrm{k}$-points is employed. For the sake of accuracy, a double k-point mesh is also tested. The difference of the total energy is less than 0.01 $\mathrm{eV}$ in the two cases. Whereas for all the density of states (DOS) calculations shown in this work, the double k-point meshes are employed. The Bader charge analysis [37] has been used in the later discussions. 
The adsorption energy is calculated as

$$
E_{a d s}=E_{t o t}(X / f i l m)-\left[E_{t o t}(f i l m)+E_{t o t}(X)\right]
$$

where $E_{\text {tot }}(X / f i l m)$ is the total energy of the slab covered with the adsorbate $X$ in its ground state, $E_{t o t}(f i l m)$ is the total energy of the bare substrate, and $E_{\text {tot }}(X)$ represents the total energy of the free adsorbate. By this definition, the more negative the $E_{a d s}$ is, the stronger the adsorption is.

\section{Results and discussion}

\subsection{Clean $\alpha-\mathrm{Fe}_{2} \mathrm{O}_{3}(0001)$ film}

To investigate the stability of the films with two different types of termination, as shown in Fig. 1 (a) and (b), the total energies of each slab with various possible anti-ferromagnetic (AFM) arrangements and a ferromagnetic (FM) arrangement have been calculated. It is found that for both type A and type B films, after structural optimization, the total energy of the slab with an AFM phase is always lower than that with an FM one. The results also show that the total energy of type B film is $0.83 \mathrm{eV}$ per formula unit lower than that of type A film by comparing their most stable configurations. It means that type B film with both sides terminated by Fe atoms is the most energetically stable film in the 12-layer film models. Cleaving energy per formula unit $\left(E_{c}\right)$ of the films with respect to the bulk structure of hematite is calculated as,

$$
E_{c}=\frac{E_{F i l m}}{n}-\frac{E_{B u l k}}{m},
$$

where $E_{\text {Film }}$ is the ground state total energy of the film and $E_{B u l k}$ is that of the bulk hematite structure. $n$ and $m$ are the number of formula units in the super cells for each 
case used in the calculations. $E_{c}$ for type B and type A films are 0.81 and $1.64 \mathrm{eV}$ per formula unit, respectively, which indicates that type B film is more energetically favorable to synthesize than type A film. Therefore, the type B film is adopted in this study.

The results of the various magnetic arrangements of type B are shown in Table 1, including FM and all possible AFM configurations. And with the AFM-4 configuration, the film has the lowest total energy and almost zero net magnetic moment. At the same time the lattice parameter $a$ is also optimized, which is $\sim 3.0 \%$ larger than its value of the bulk structure. Hence, it is understandable to find that the length of all of the Fe-O bonds is increased by $2.1 \%-2.4 \%$ except those of the terminated $\mathrm{Fe}$ atoms with their neighboring $\mathrm{O}$ atoms. The bond length of the terminated Fe's with the underneath $\mathrm{O}$ atoms is decreased by $\sim 6.5 \%$. In the following studies, the most stable type B film (AFM-4) with the optimized lattice constant will be used.

The density of states (DOS) of the film is shown in Fig. 2, together with that of the bulk hematite for comparison. There are some differences between the film and the bulk in Fig. 2. The DOS of the film between spin-up and spin-down is asymmetric. This is due to the asymmetrical atomic magnetic moment arrangement, i.e. AFM-4 in Table 1, resulting from that both sides are terminated by the spin-up $\mathrm{Fe}$ atoms. Obviously, there are new spin-down states at the energy level at about $1.43 \mathrm{eV}$ above the valance band minimum (VBM). Due to the new states, though unoccupied, the energy band gap is dicreased from $\sim 2.05 \mathrm{eV}$ of the bulk to $\sim 1.43 \mathrm{eV}$ for the film. The 
new surface states result from the Fe-3d states of the terminated Fe atoms. In addition, there is a broken symmetry for spin up and down in the valence bands of the film. The DOS from $-5.0 \mathrm{eV}$ to $0.0 \mathrm{eV}$ are shifted between the spin-up states and the spin-down states. Fig. 2 also shows that the VBM is dominated mainly by O-2p states, while the lowest conduction band edge is primarily $\mathrm{Fe}-3 \mathrm{~d}$ states in character, similar to the situation of the bulk. The magnetic moment of the terminated Fe atoms on the surface is $\sim 4.02 \mu_{\mathrm{B}}$, discreased by $3.6 \%$ from the value of the inner Fe atoms. But the film still keeps the AFM orientation.

\subsection{S-adsorption on the $\alpha-\mathrm{Fe}_{2} \mathrm{O}_{3}(0001)$ surface}

According to the previous theoretical study, the isovalent doping of $\mathrm{S}$ in the bulk $\alpha-\mathrm{Fe}_{2} \mathrm{O}_{3}$ can modify significantly the band edges near the band gap [24]. It is important to understand the feasibility of the incorporation of sulfur into $\alpha-\mathrm{Fe}_{2} \mathrm{O}_{3}$. The initial stage of the doping process could be the adsorption of $\mathrm{S}$ on the $\alpha-\mathrm{Fe}_{2} \mathrm{O}_{3}$ surface. For an $\mathrm{S}$ adatom adsorption on the suface of the film model discussed in the previous section, various sites have been examined. They are top (T) sites of the Fe or O atoms, hollow (H) sites and several bridge (B) sites, as shown in Fig. 3.

There are three Fe atom layers and one $\mathrm{O}$ atom layer are shown for S-adsorption in Fig. 3. The top-most Fe atom layer is named as Fe(I) (blue), the second Fe atom layer is called as $\mathrm{Fe}$ (II ) (purple), and the third Fe atom layer is labeled as $\mathrm{Fe}(\mathrm{III})$ (cyan). The $\mathrm{O}$ atom layer is between the $\mathrm{Fe}(\mathrm{I})$ layer and $\mathrm{Fe}(\mathrm{II})$ layer. $\mathrm{T} 1$ and $\mathrm{T} 2$ are the top sites of the $\mathrm{Fe}(\mathrm{I})$ and $\mathrm{O}$ atom, respectively. $\mathrm{H} 1, \mathrm{H} 2$, and $\mathrm{H} 3$ are three hollow sites of $\mathrm{O}$ atoms which form the equilateral triangle, but the bottom atoms under the hollow sites 
are the $\mathrm{Fe}$ ( II ), the $\mathrm{Fe}$ (III) and an $\mathrm{O}$ atom from even deeper layer, respetively. B1, B2, and $\mathrm{B} 3$ are three bridge sites of $\mathrm{O}$ atoms. These bridge sites are across two neighboring oxygen atom in the same layer. $\mathrm{B} 4$ is a bridge site between the bonded $\mathrm{O}$ atom and $\mathrm{Fe}(\mathrm{I})$ atom. Table 2 lists only the stable sites at which $\mathrm{S}$ atom does not relax away from the initial position after the optimization. In Table 2, the relative energy is measured from one of the most stable site, which is $\mathrm{H} 2$.

Fig. 4 gives the side view of the S-adsorption on the most stable site on the surface. The adsorption energy of the most stable adsorption site is calculated as $-4.20 \mathrm{eV}$ using Eq. (1). As listed in the second row of the Table 2, for the most stable adsorption site (H2), the nearest neighbors of the adsorbate sulfur atom are the three oxygen atoms, forming an equilateral triangle, with the S-O distance of $1.56 \AA$. As a result, the three $\mathrm{O}$ atoms move upwards by about $0.49 \AA$. The distance between the adsorbate $\mathrm{S}$ atom and the second nearest neighbor, which is the Fe (I) atom on the first layer, is much larger, about $3.18 \AA$.

Surprisingly, the $\mathrm{S}$ adatom prefers to bond with the three $\mathrm{O}$ atoms on the surface, in the centre of the equilateral triangle formed by the three $\mathrm{O}$ atoms. In other words, the $\mathrm{S}$ atom would like to form a $\mathrm{SO}_{3}$ cluster with the three $\mathrm{O}$ atoms on the surface. After the adsorption of sulfur atom, due to the bonding of the three $\mathrm{O}$ atoms and the $\mathrm{S}$ adatom, the original bonds between the three $\mathrm{O}$ atoms and the underneath $\mathrm{Fe}$ atom, $\mathrm{Fe}$ (III), are broken, causing a significant downward displacement of the Fe (III) by $\sim 1.95 \AA$. And the magnetic moment of the $\mathrm{Fe}$ (I) atom which bonds with the $\mathrm{O}$ atoms in $\mathrm{SO}_{3}$ is decreased by $13.6 \%$. 
In Fig. 5, the total and projected DOS for the different atoms on the most stable site of the film are presented. The top of the valence bands and the bottom of the conduction bands are dominated mainly by $\mathrm{O}-2 \mathrm{p}$ states and Fe-3d states, respectively, which is unchanged from that of the clean film. Comparing with the DOS of the clean film, the $\mathrm{S}$ adsorption induces two additional gap states in the band gap discussed in section 3.1. They are not directly coming from the $\mathrm{S}$ atom. The main contribution of the states in the band gap is from the Fe atoms at the nearby of the $\mathrm{S}$ adatom. According to the analysis of the projected DOS of each atom, which is not shown here, the lower spin-down peak is contributed by the three second nearest neighbor Fe (I) atoms shown in Fig. 4. And the spin-up peak is contributed by the Fe (III) atom which has a significant downward displacement as shown in Fig. 4.

It is also important to understand the migration of the $\mathrm{S}$ adatom on the hematite $\alpha-\mathrm{Fe}_{2} \mathrm{O}_{3}(0001)$ film. It is found that the $\mathrm{S}$ atom remains to bond with the three $\mathrm{O}$ atoms after it is placed beneath the plane of the oxygen triangle. The total energy of the configuration after geometry optimization is $0.37 \mathrm{eV}$ higher than that of the most stable S-adsorption configuration. The diffusion of the $\mathrm{S}$ adatom is evaluated and the transition state along the minimum-energy pathway is searched using the climbing-image nudged elastic band (CI-NEB) method. As shown in Fig. 6, the most stable S-adsorption configuration and the configuration described above are selected as the initial state and the final state. Obviously, the diffusion goes through a barrier of energy from the initial state to the final state. The pathway and the transition state are also shown in Fig. 6 and the energy barrier is about $1.98 \mathrm{eV}$. In Fig. 6, for the 
transition state, the sulfur atom is co-plane with the three $\mathrm{O}$ atom triangle. But due to the larger size of sulfer atom, one oxygen atom is pushed a little further than the other two $\mathrm{O}$ atoms.

\subsection{S-doping in the $\alpha-\mathrm{Fe}_{2} \mathrm{O}_{3}(0001)$ film}

To study the sulfur atom doping in the film, only the $\mathrm{S}$ substitution of $\mathrm{O}$ atom is considered. For our hematite film model, as shown in Fig. 1 (b), there are four oxygen layers, with a symmetry. The $\mathrm{O}$ atoms in the fourth oxygen layer have the same local environment, such as the nearest neighbors and the corresponding bond lengths, as those of the first oxygen layer. Similarly, the $\mathrm{O}$ atoms in the third oxygen layer and the second oxygen layer have the same local symmetry. For the study of the sulfur doping in the film, the substitutions of oxygen atom on the first oxygen layer and the second oxygen layer are considered. The $\mathrm{S}$ atom substitution of the first layer $\mathrm{O}$ atom is energetically $0.56 \mathrm{eV}$ lower than that of the substitution of the second layer $\mathrm{O}$ atom. In other words, it is more stable to substitute of the sulfur atom for the surface oxygen atom. This indicates that $\mathrm{S}$ atom prefers to stay at the surface. The total and projected DOS of the S-doping in the first oxygen layer is given in the Fig. 7. It shows that the band gap is reduced to $\sim 1.26 \mathrm{eV}$ from $\sim 1.43 \mathrm{eV}$ of the clean film, similar to the S-doping in bulk $\mathrm{Fe}_{2} \mathrm{O}_{3}$ [24]. The maximum of the valence band is dominated by $\mathrm{O}-2 \mathrm{p}$ and S-3p states, while the bottom of the conduction bands is mainly contributed by Fe-3d states, similar to S-doping in the bulk.

The feasibility of the isovalent $\mathrm{S}$ substitution in the film of the hematite $\alpha-\mathrm{Fe}_{2} \mathrm{O}_{3}$ has been investigated by calculating the formation energy according to the formula 


$$
E_{\text {form }}=E_{\text {tot }}(\text { doped film })-E_{\text {tot }}(\text { clean film })-\mu_{S}+\mu_{O}
$$

where $E_{\text {tot }}$ (doped film) and $E_{\text {tot }}($ clean film) are the total energies of the S-doped and the clean hematite slab, respectively. $\mu_{S}$ and $\mu_{O}$ denote the chemical potentials of sulfur and oxygen atom, respectively. The $\mu_{O}$ can be obtained from the

ground-state total energy of the $\mathrm{O}_{2}$ molecule $\left(\mu_{O}=1 / 2 \mu_{\left(O_{2}\right)}\right)$, while the $\mu_{S}$ is calculated from the $\alpha-S_{8}$. The calculated formation energies for the first and second layer are $2.29 \mathrm{eV}$ and $2.85 \mathrm{eV}$ per sulfur substitution, respectively. So it is relatively hard for $\mathrm{S}$ to be doped into the clean $\mathrm{Fe}_{2} \mathrm{O}_{3}$ film.

For the S-adsorption and the isovalent S-doping, the Bader charge analysis has been applied. It is found that the Bader partial charge of the $\mathrm{S}$ ion is $\sim 3.65 \mathrm{e}$ and $\sim 6.67 \mathrm{e}$ for S-adsorption and S-doping, respectively. It means that the $\mathrm{S}$ acts as a cation in the S-adsorption position, while it presents anionic behavior in the S-doping condition.

\section{Conclusions}

In summary, the stability of the clean hematite $\alpha-\mathrm{Fe}_{2} \mathrm{O}_{3}$ (0001) film built of 12 atomic layers with two different types of termination has been studied by DFT $+U$ method. The film terminated by Fe atoms on both sides with the AFM arrangement is the most stable one. For a sulfur adsorption on the suface of this model, various sites have been examined. It is found that the $\mathrm{S}$ adatom prefers to bond with the three $\mathrm{O}$ atoms on the second layer of the surface, in the centre of the equilateral triangle formed by the three $\mathrm{O}$ atoms. Comparing with the DOS of the clean film, there are two gap states in the band gap due to the adsorption, in addition to the original surface 
states. The $\mathrm{S}$ adatom acts as a cation here, and has no direct contribution to the gap states of the film electronic structure. Meanwhile, the $\mathrm{S}$ atom substitution of the first layer $\mathrm{O}$ atom is energetically $0.56 \mathrm{eV}$ lower than that of the substitution of the second layer $\mathrm{O}$ atom. From the study of the electronic properties of the S-doping in the film, it shows that the band gap is reduced to $\sim 1.26 \mathrm{eV}$ from $\sim 1.43 \mathrm{eV}$ of the clean film, similar to the S-doping in bulk $\mathrm{Fe}_{2} \mathrm{O}_{3}$.

The results of both the adsorption and the substitution by $\mathrm{S}$ atom at the $\alpha-\mathrm{Fe}_{2} \mathrm{O}_{3}$ (0001) surface suggest that $S$ atom prefers to stay at the surface and it could be difficult to be doped into the material and modify the band gap of $\alpha-\mathrm{Fe}_{2} \mathrm{O}_{3}$ as previous expected. This excludes the possibility of tuning the band gap of $\alpha-\mathrm{Fe}_{2} \mathrm{O}_{3}$ through the sulfurization of the oxide surfaces, followed by thermal diffusions. Therefore, the doping of $\mathrm{S}$ in $\alpha-\mathrm{Fe}_{2} \mathrm{O}_{3}$ should be investigated by alternative ways, such as ion implementation followed by thermal annealing.

\section{Acknowledgements}

This research was supported by NSF SusChEM Program (Award No. DMR-1306291 and DMR-1306542). The computational work was done at the High Performance Computing Center of the University of Texas at Arlington and the Texas Advanced Computing Centre (TACC) at the University of Texas at Austin.

\section{References}

[1] Teja A.S., Koh, P.Y., Prog. Cryst. Growth Charact. Mater. 2009, 55, 22-45.

[2] Mahmoudi M., Simchi I., Imani M., J. Iran. Chem. Soc., 2010, 7, S1-S27.

[3] Wang G., Liu T., Luo Y., Zhao Y., Ren Z., Bai, J., Wang H., J. Alloys Compd., 
2011, 509, L216-L220.

[4] Gupta A.K., Gupta M., Biomaterials 2005, 26, 3995-4021.

[5] Larry W. Finger and Robert M. Hazen, J. Appl. Phys., 1980, 51, 5362-5367.

[6] J. Staun Olsen, C. S. G. Cousins, L. Gerward, H. Jhans and B. J. Sheldon, Physica Scripta, 1991, 43, 327-330.

[7] F. J. Morin, Phys. Rev., 1953, 93, 1195-1199.

[8] C. Gieitzer, J. Nowotny and M. Rekas, Appl. Phys. A, 1991, 53, 310-316.

[9] G. Rollmann, A. Rohrbach, P. Entel, J. Hafner, Phys. Rev. B, 2004, 69, 165107.

[10] A. H. Hill, F. Jiao, P. G. Bruce, A. Harrison, W. Kockelmann, and C. Ritter, Chem. Mater., 2008, 20, 4891-4899.

[11] Carlos J Serna, Manuel Ocafia and Juan E Iglesias, J. Phys. C: Solid State Phys., $1987,20,473-484$.

[12] Tadanori Hashimoto, Tetsuya Yamada, and Toshinobu Yoko, J. Appl. Phys., 1996, $80,3184-3190$.

[13] Kevin Sivula, Florian Le Formal, and Michael Grätzel, ChemSusChem, 2011, 4, 432-449.

[14] Yuzheng Guo, Stewart J Clark, and John Robertson, J. Phys.: Condens. Matter, $2012,24,325504$.

[15] Alan Kleiman-Shwarsctein, Yong-Sheng Hu, Arnold J. Forman, Galen D. Stucky, and Eric W. McFarland, J. Phys. Chem. C, 2008, 112, 15900-15907.

[16] Ilkay Cesar, Andreas Kay, José A. Gonzalez Martinez, and Michael Grätzel, J. Am. Chem. Soc., 2006, 128, 4582-4583.

[17] Yong-Sheng Hu, Alan Kleiman-Shwarsctein, Arnold J. Forman, Daniel Hazen, Jung-Nam Park, and Eric W. McFarland, Chem. Mater., 2008, 20, 3803-3805.

[18] Omid Zandi, Benjamin M. Klahr and Thomas W. Hamann, Energy Environ. Sci., $2013,6,634-642$.

[19] Peilin Liao, John A. Keith, and Emily A. Carter, J. Am. Chem. Soc., 2012, 134, 13296-13309.

[20] Nerine J. Cherepy, Dorion B. Liston, Jennifer A. Lovejoy, Hongmei Deng, and Jin Z. Zhang, J. Phys. Chem. B, 1998, 102, 770-776. 
[21] Alan Kleiman-Shwarsctein, Muhammad N. Huda, Aron Walsh, Yanfa Yan, Galen D. Stucky, Yong-Sheng Hu, Mowafak M. Al-Jassim, and Eric W. McFarland, Chem. Mater., 2010, 22, 510-517.

[22] T. Droubay, K. M. Rosso, S. M. Heald, D. E. McCready, C. M. Wang, and S. A. Chambers, Phys. Rev. B, 2007, 75, 104412.

[23] X. Y. Meng, G. W. Qin, S. Li, X. H. Wen, Y. P. Ren, W. L. Pei and L. Zuo, Appl. Phys. Lett., 2011, 98, 112104.

[24] Congxin Xia, Yu Jia, Meng Tao, and Qiming Zhang, Physics Letters A, 2013, 377, 1943-1947.

[25] Wolfgang Bergermayer and Hannes Schweiger, Phys. Rev. B, 2004, 69, 195409.

[26] Manh-Thuong Nguyen and Ralph Gebauer, J. Phys. Chem. C, 2014, 118, 8455-8461.

[27] Jiajia Song, Xiaoqi Niu, Lixia Ling, Baojun Wang, Fuel Processing Technology, 2013, 115, 26-33.

[28] Frank J. Berry, Alberto Bohorquez, Colin Greaves, Julia McManus, Elaine A. Moore, Michael Mortimer, J. Solid State Chem., 1998, 140, 428-430.

[29] Adam Kiejna and Tomasz Pabisiak, J. Phys.: Condens. Matter, 2012, 24, 095003.

[30] Kresse G and Hafner J, Phys. Rev. B, 1993, 47, 558.

[31] Kresse G and Furthmüller J, Phys. Rev. B, 1996, 54, 11169.

[32] J.P. Perdew, J.A. Chevary, S.H. Vosko, K.A. Jackson, M.R. Pederson, D.J. Singh, C. Fiolhais, Phys. Rev. B, 1992, 46, 6671-6687.

[33] Kresse G and Joubert D, Phys. Rev. B, 1999, 59, 1758.

[34] Dudarev S L, Botton G A, Savrasov S Y, Humphreys C J and Sutton A P, Phys. Rev. B, 1998, 57,1505.

[35] Graeme Henkelman, Blas P. Uberuaga and Hannes Jónsson, J. Chem. Phys., 2000, 113, 9901.

[36] Hendrik J. Monkhorst and James D. Pack, Phys. Rev. B, 1976, 13, 5188.

[37] Graeme Henkelman, Andri Arnaldsson, and Hannes Jónsson, Computational Materials Science, 2006, 36, 354-360. 


\section{Figure captions}

Fig. 1. The $2 \times 2 \times 1$ hematite (0001) surface. (a) is the film of type A. (b) is the film of type $\mathrm{B}$. The Fe atoms are blue large balls and the $\mathrm{O}$ atoms are red small ones.

Fig. 2. The density of states (DOS) of the bulk hematite (a) and the (0001) film (b). The positive and negative value represent spin up and spin down, respectively. The zero energy is the valence band maximum (VBM).

Fig.3. The top view of various sites for S-adsorption. The small balls are $\mathrm{O}$ atoms below the top layer. All the big balls are Fe atoms. The blue color is the top layer Fe's. The purple color is the second layer Fe's. The cyan color is the third layer Fe's. The lowest-energy adsorption site for $\mathrm{S}$ is $\mathrm{H} 2$.

Fig. 4. The side view of the most stable site for S-adsorption. The bigger balls are Fe (I) (blue), Fe (II) (purple), and Fe (III) (cyan) atoms. The smaller balls are $\mathrm{O}$ atoms. The middle size ball is the $\mathrm{S}$ adatom.

Fig. 5. The density of states (DOS) of the S-adorption on hematite $\alpha-\mathrm{Fe}_{2} \mathrm{O}_{3}(0001)$ film: the total DOS of the film with the S adsorbed (a); the projected DOS of the Fe and $\mathrm{O}$ atoms (b); the projected DOS of the $\mathrm{S}$ adatom (c). The zero energy is aligned with the clean film with respect to 1 s core level of a remote $\mathrm{O}$ atom.

Fig. 6. The schematic potential energy profiles for the $\mathrm{S}$ atom diffusion on the hematite (0001) film. The side views of structures of the initial state, transition state and final state are given.

Fig. 7. The density of states (DOS) of the S-doing in the first oxygen layer of hematite $\alpha-\mathrm{Fe}_{2} \mathrm{O}_{3}(0001)$ film: the total DOS (a); the projected DOS for the Fe and $\mathrm{O}$ atoms (b); 
the projected DOS for the S atom(c). The zero energy is aligned with the clean film with respect to $1 \mathrm{~s}$ core level of a remote $\mathrm{O}$ atom. 


\section{Tables}

Table. 1. The different possible magnetic arrangements of type B film compared by the relative total energies and the magnetization moments. A symbol $\uparrow$ or $\downarrow$ indicates the orientation of total magnetic moments of each Fe atomic layer.

\begin{tabular}{c|c|c|c}
\hline Configurations & $\begin{array}{c}\text { Magnetic } \\
\text { arrangement of iron } \\
\text { layers }\end{array}$ & $\begin{array}{c}\text { Relative total } \\
\text { energy per } \\
\text { formula unit } \Delta E_{\text {tot }} \\
(\mathrm{eV})\end{array}$ & $\begin{array}{c}\text { Total } \\
\text { magnetization } \\
\text { per formula unit } \\
\left(\mu_{B}\right)\end{array}$ \\
\hline AFM-1 & $\uparrow \uparrow \downarrow \downarrow \downarrow \downarrow \uparrow \uparrow$ & 0.32 & -0.05 \\
\hline AFM-2 & $\uparrow \uparrow \downarrow \downarrow \uparrow \uparrow \downarrow \downarrow$ & 0.27 & 0.00 \\
\hline AFM-3 & $\uparrow \uparrow \uparrow \uparrow \downarrow \downarrow \downarrow \downarrow$ & 0.51 & 0.00 \\
\hline AFM-4 & $\uparrow \downarrow \downarrow \uparrow \uparrow \downarrow \downarrow \uparrow$ & 0.00 & 0.00 \\
\hline AFM-5 & $\uparrow \downarrow \uparrow \downarrow \uparrow \downarrow \uparrow \downarrow$ & 0.25 & 9.67 \\
\hline FM & $\uparrow \uparrow \uparrow \uparrow \uparrow \uparrow \uparrow \uparrow$ & 0.61 & \\
\hline
\end{tabular}

Table 2. The adsorption sites comparison with the relative energy $\Delta \mathrm{E}_{\text {tot }}$, the nearest neighbors of the adsorbate atom and their distance after the geometry optimization.

\begin{tabular}{c|c|c|c|c|c}
\hline Site & $\Delta \mathrm{E}_{\text {tot }}(\mathrm{eV})$ & \multicolumn{2}{|c|}{$\begin{array}{c}\text { First nearest neighbors } \\
\text { and the distance }(\AA)\end{array}$} & \multicolumn{2}{|c}{$\begin{array}{r}\text { Second nearest } \\
\text { neighbors and the } \\
\text { distance }(\AA)\end{array}$} \\
\hline $\mathrm{T} 1$ & 1.87 & $\mathrm{Fe}$ & 2.28 & $\mathrm{O}$ & 3.44 \\
\hline $\mathrm{H} 2$ & 0.00 & $\mathrm{O}$ & 1.56 & $\mathrm{Fe}$ & 3.18 \\
\hline $\mathrm{T} 2$ & 0.85 & $\mathrm{O}$ & 1.74 & $\mathrm{Fe}$ & 2.26 \\
\hline $\mathrm{B} 3$ & 1.005 & $\mathrm{O}$ & 1.64 & $\mathrm{Fe}$ & 2.50 \\
\hline $\mathrm{H} 3$ & 2.38 & $\mathrm{O}$ & 1.57 & $\mathrm{Fe}$ & 2.52 \\
\hline
\end{tabular}


Fig. 1
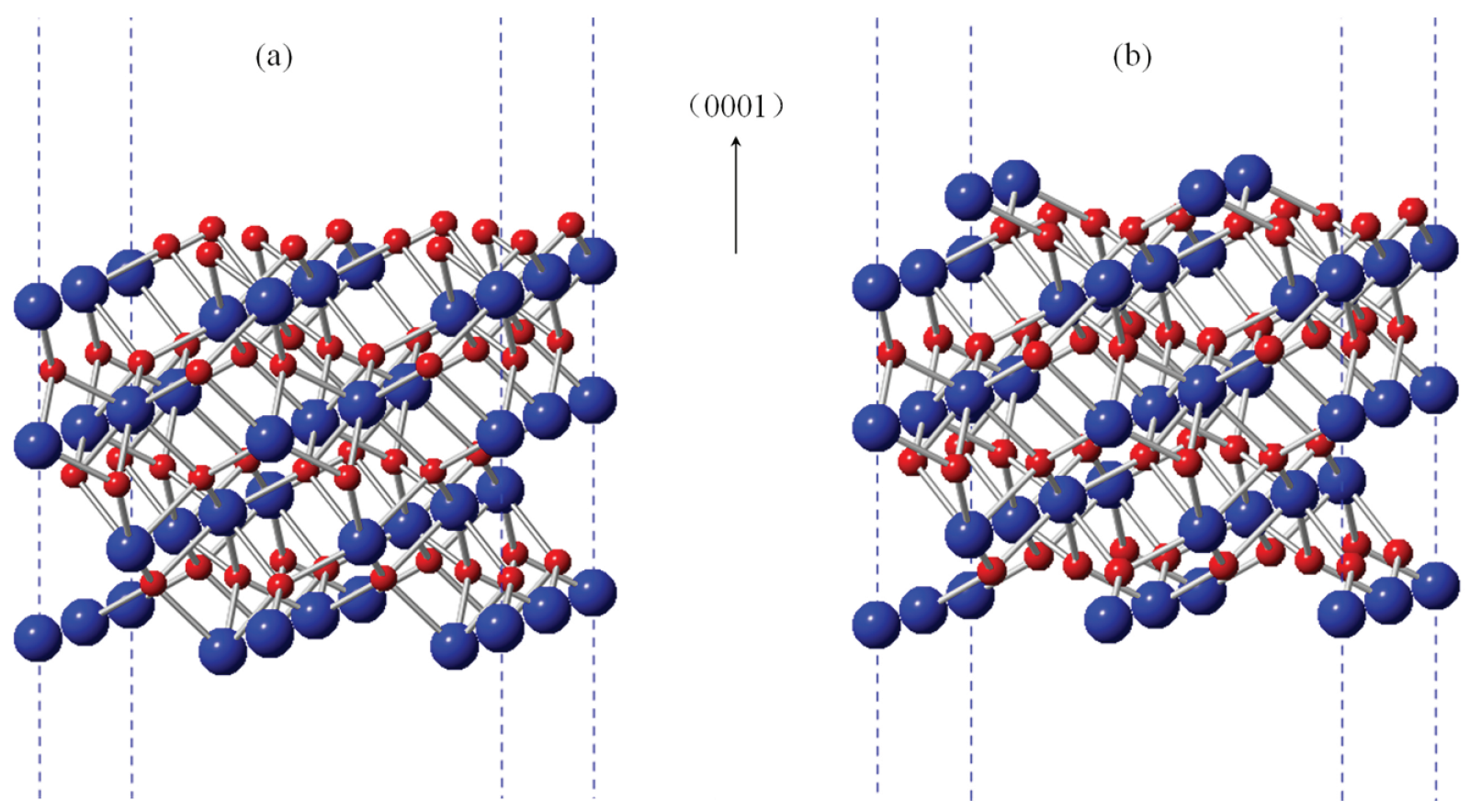
Fig. 2

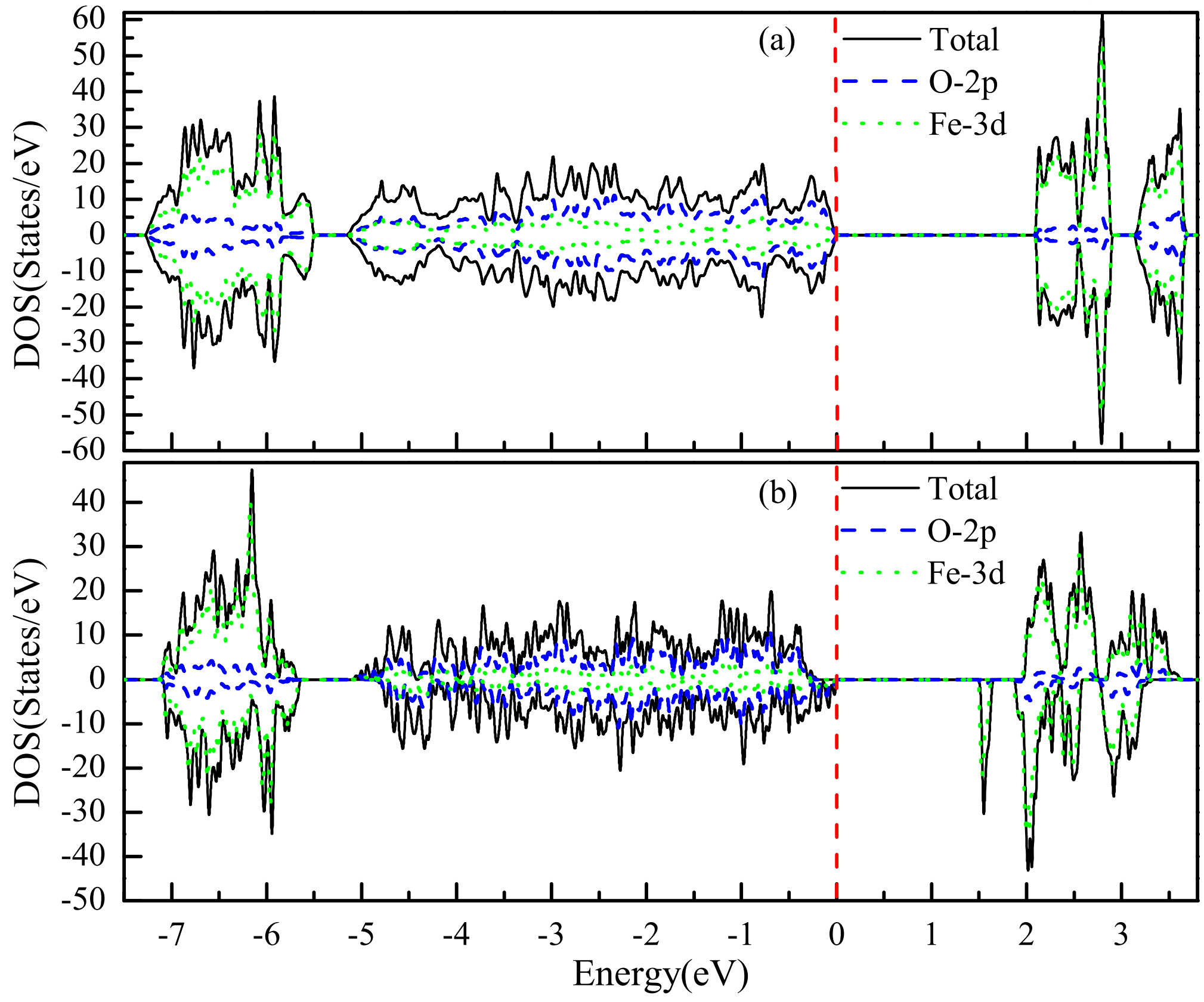


Fig. 3

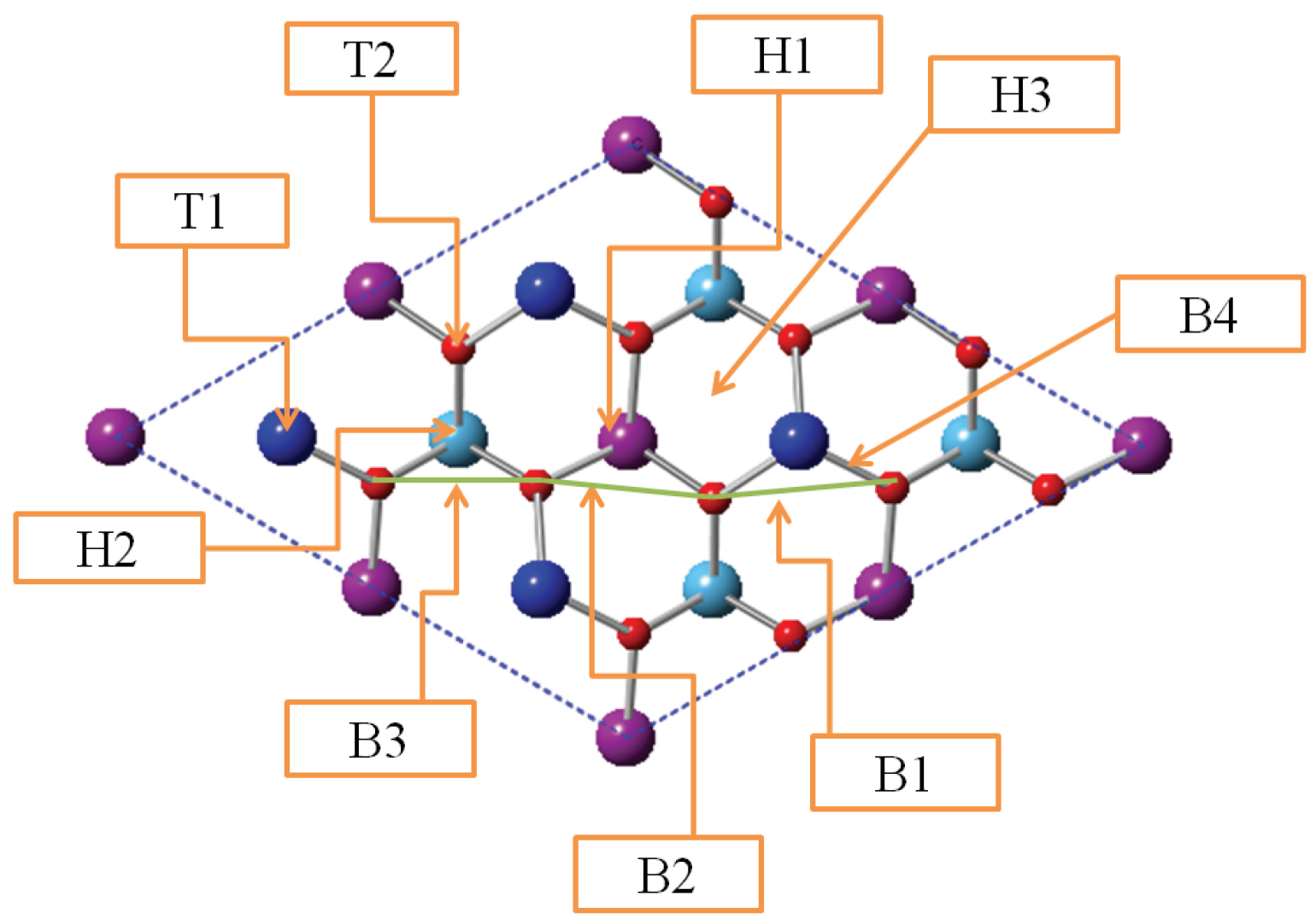


Fig. 4

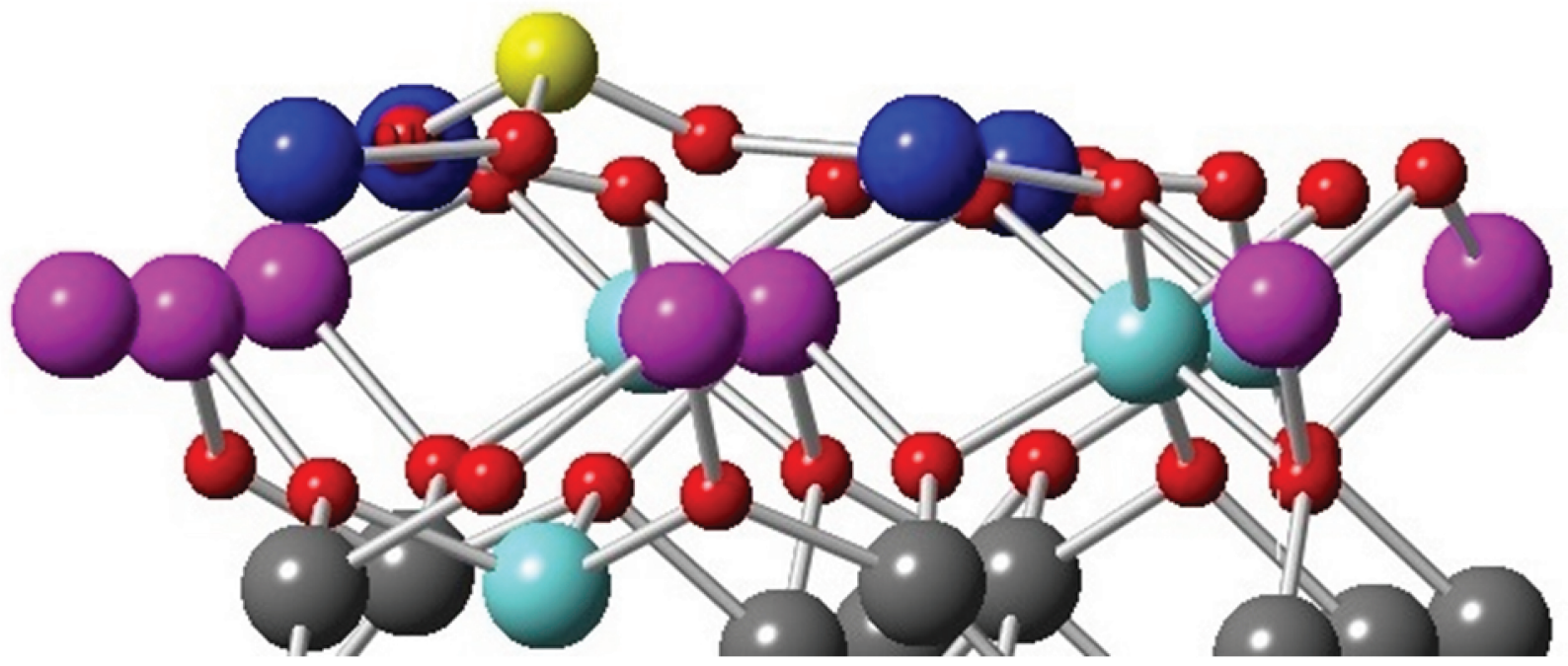


Fig. 5

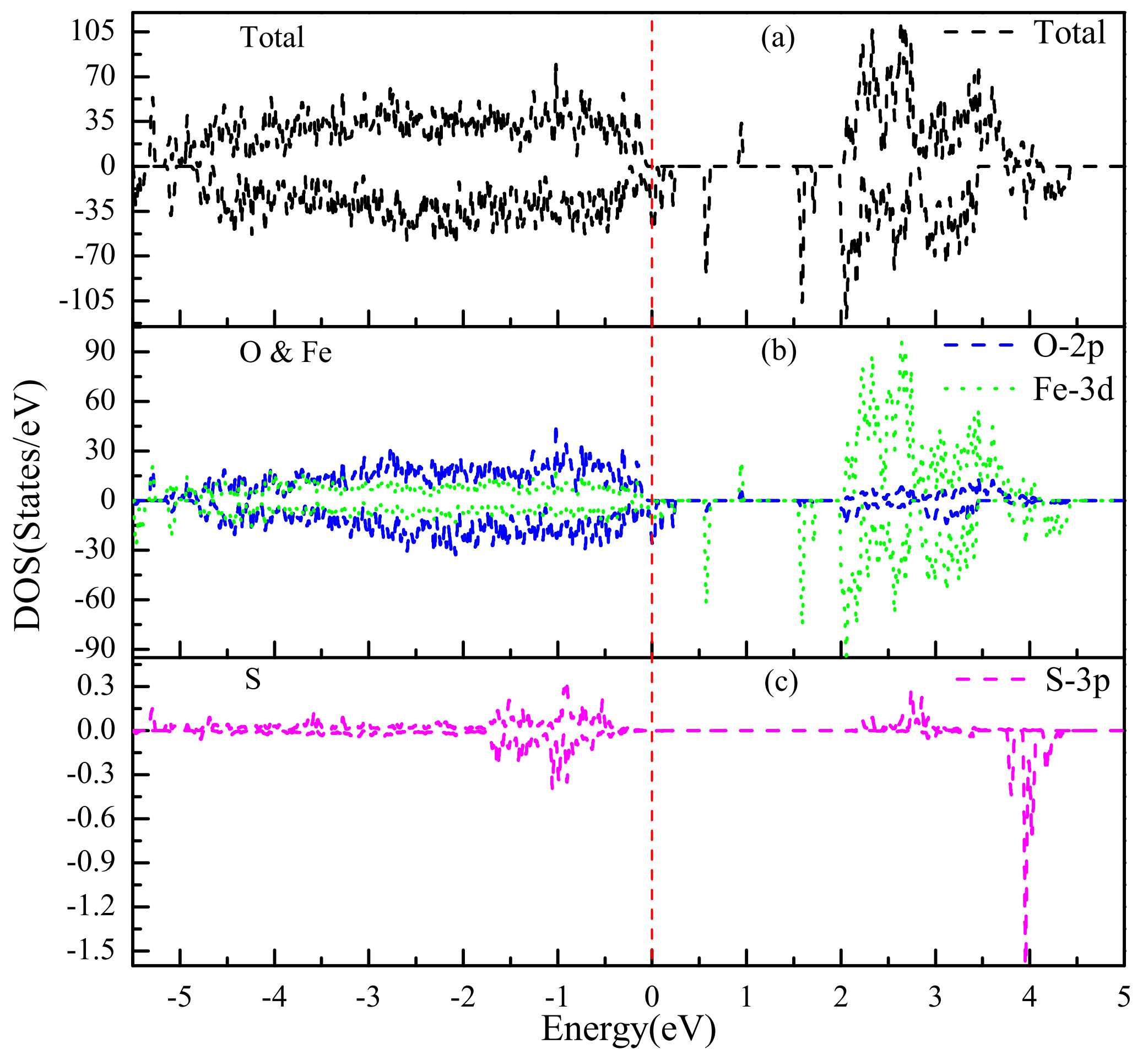


Fig. 6

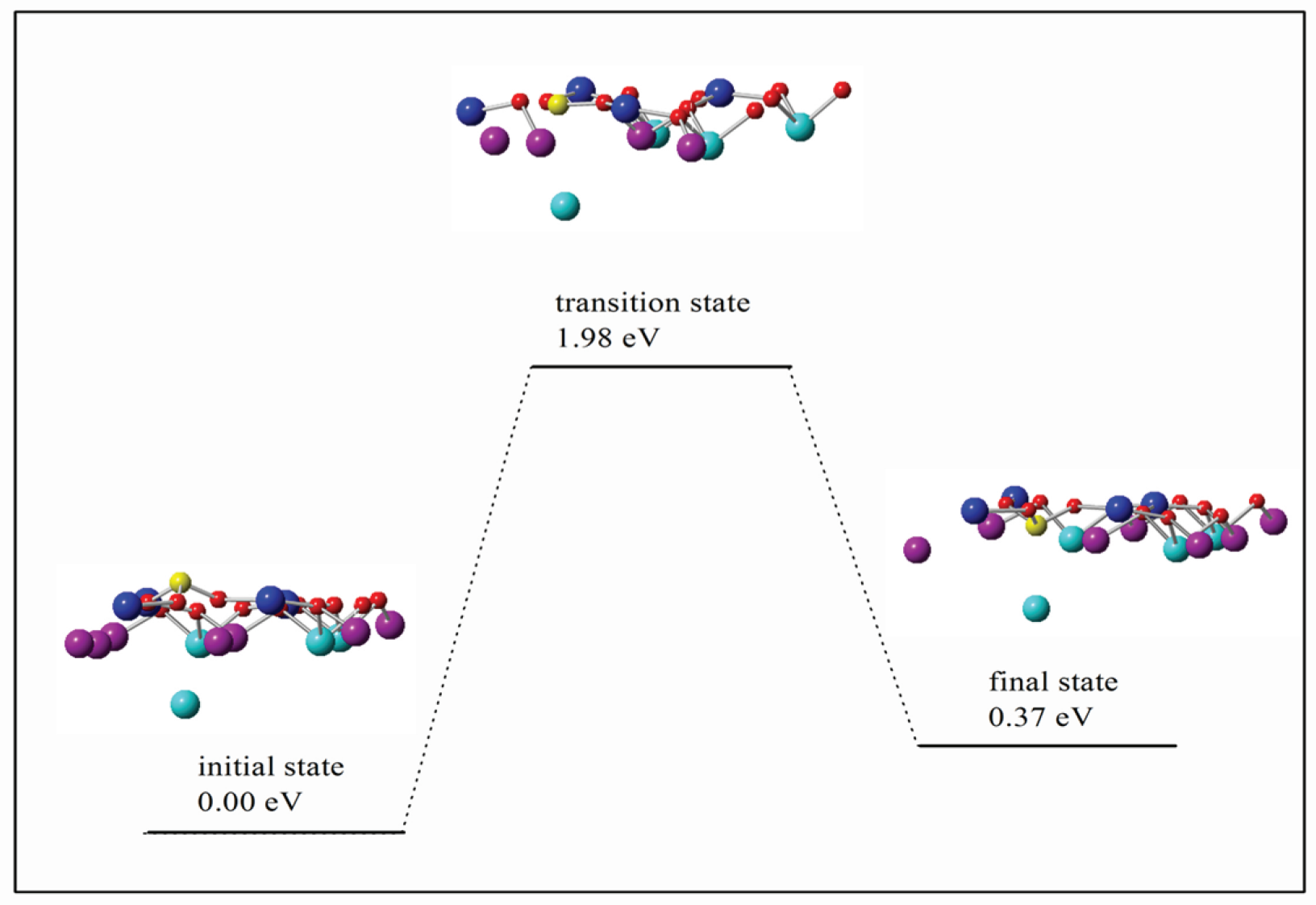


Fig.7

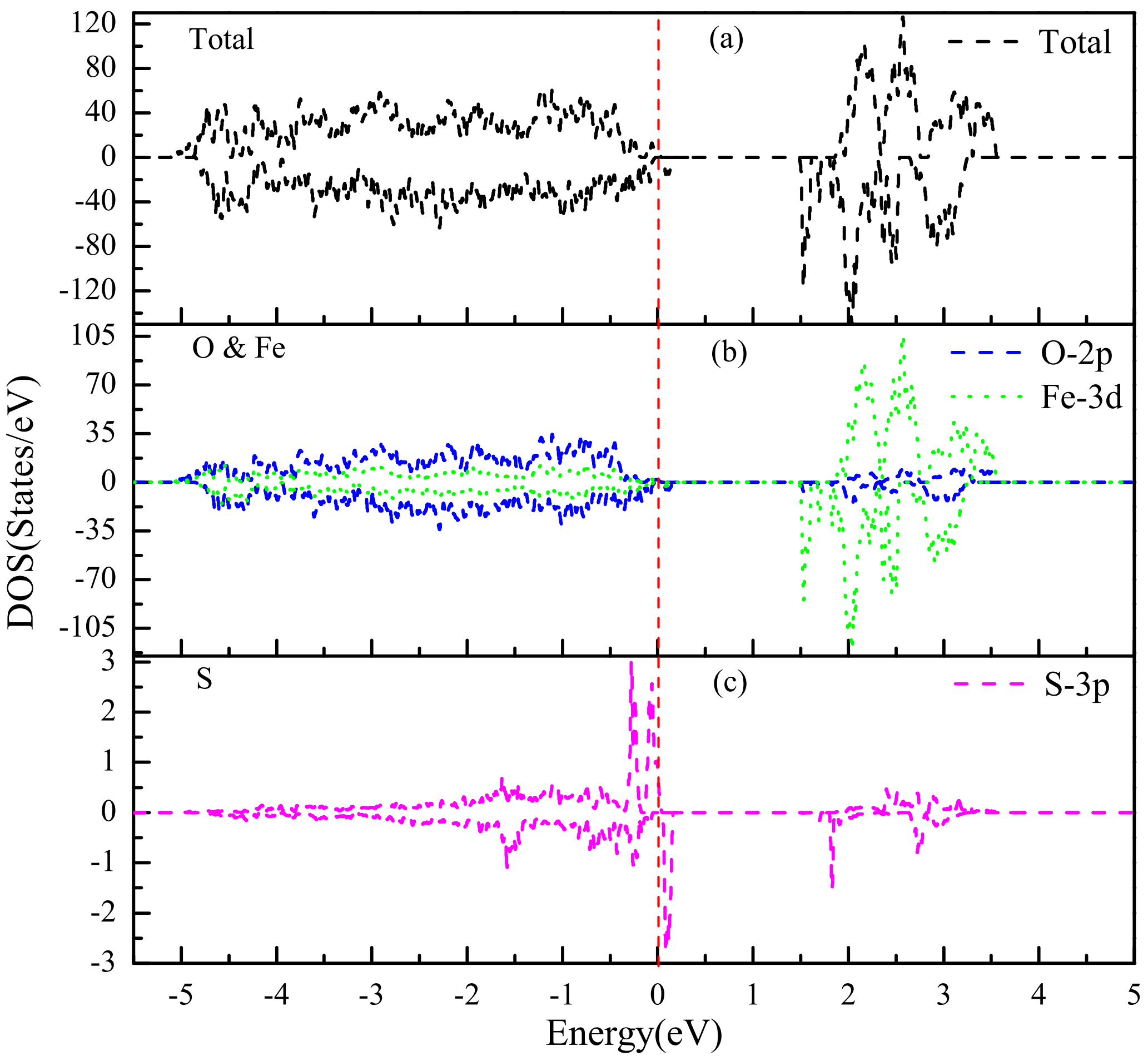

